\title{
On Characteristic Exponents in Turbulence
}

\author{
Elliott H. Lieb* \\ Departments of Mathematics and Physics, Princeton University, P.O. Box 708, Princeton, NJ 08544, \\ USA
}

\begin{abstract}
Ruelle has found upper bounds to the magnitude and to the number of non-negative characteristic exponents for the Navier-Stokes flow of an incompressible fluid in a domain $\Omega$. The latter is particularly important because it yields an upper bound to the Hausdorff dimension of attracting sets. However, Ruelle's bound on the number has three deficiences: (i) it relies on some unproved conjectures about certain constants; (ii) it is valid only in dimensions $\geqq 3$ and not 2 ; (iii) it is valid only in the limit $\Omega \rightarrow \infty$. In this paper these deficiences are remedied and, in addition, the final constants in the inequality are improved.
\end{abstract}

Ruelle [1] has derived upper bounds on the magnitude and number of nonnegative characteristic exponents of the Navier-Stokes equation for the flow of an incompressible fluid in a domain $\Omega \in \mathbb{R}^{d}$. The bound on the number, $\tilde{N}(\mu)$ [defined in (42)], is particularly interesting because it leads to an upper bound on the Hausdorff dimension of a compact attracting set [1, Corollary 2.3]. Unfortunately, the bounds in [1] on $\tilde{N}(\mu)$, unlike those on the magnitude, have certain deficiencies which are

(i) They rely for their validity on some conjectured, but as yet unproved, relations between the sharp constants in two known inequalities.

(ii) They are valid only for $d \geqq 3$.

(iii) Because Weyl's asymptotic formula for the eigenvalues of the Laplacian in $\Omega$ is used, the inequalities are not valid for any fixed $\Omega$, but only in the limit $\Omega \rightarrow \infty$.

In this paper a different proof of Ruelle's inequality for the number will be given so that the above three deficiencies are remedied. The result is contained in Eqs. (40)-(43).

Let $v: \Omega \rightarrow \mathbb{R}^{d}$ denote a solution to the Navier-Stokes equation, and let $\mu_{1} \geqq \mu_{2} \geqq \ldots$ be the characteristic exponents corresponding to a probability measure $\varrho(d v)$ on the space of solutions that is ergodic with respect to the Navier-

\footnotetext{
* Work partially supported by U.S. National Science Foundation grant No. PHY-8116101-A01
} 
Stokes time evolution. Ruelle shows [1] that for all $n \geqq 1$

$$
\sum_{i=1}^{n d} \mu_{i} \leqq-d \sum_{i=1}^{n}\left\langle e_{i}\right\rangle \equiv-d\left\langle E_{n}\right\rangle \text {. }
$$

The brackets $\langle\cdot\rangle$ denote average with respect to $\varrho, E_{n}=\sum_{1}^{n} e_{i}$, and the $e_{i}=e_{i}(v)$ are ordered such that $e_{1} \leqq e_{2} \leqq \ldots$ and are the eigenvalues of the Schrödinger operator

$$
H=-v \Delta-w(x)
$$

with Dirichlet boundary conditions on $\Omega$. Here, $v$ is the kinematic viscosity and $w(x) \geqq 0$ with

$$
w(x)^{2}=[(d-1) / 4 d] \sum_{i, j}\left(\partial v_{i} / \partial x_{j}+\partial v_{j} / \partial x_{i}\right)^{2}=[(d-1) / 2 v d] \varepsilon(x) .
$$

The quantity $\varepsilon(x)$ is the rate of energy dissipation per unit mass in the flow $v$. In (2), (3) and henceforth, explicit dependence of the various quantities on $v$ is understood but not explicitly indicated unless necessary.

One might try to take additional advantage of the fact that $\operatorname{div} v=0$ but, as in [1], we shall merely assume that $w$ is some given non-negative function. It will, however, be assumed, as in [1], that

$$
w \in L^{1+d / 2}(\Omega) .
$$

Remark. The definition (3) has a factor $(d-1) / d$, which is an improvement over that in [1]. The reason is the following: Ruelle starts with an operator on $L^{2}\left(\mathbb{R}^{d}\right) \otimes \mathbb{R}^{d}$ given by $\mathscr{H}=-v \Delta \delta_{i j}+W_{i j}(x)$, where $W_{i j}(x)$ is the $d \times d$ symmetric matrix $W_{i j}(x)=\left(\partial v_{i} / \partial x_{j}+\partial v_{j} / \partial x_{i}\right) / 2$. Ruelle notes that the eigenvalues of $\mathscr{H}$ will satisfy (1) if $w(x)$ in (2) is the largest eigenvalue of the matrix $W_{i j}(x)$. This he estimates by $\left(\operatorname{Tr} W^{2}\right)^{1 / 2}$, and this leads to $(3)$ without $(d-1) / d$. Since $\operatorname{div} v=0$, however, $\operatorname{Tr} W=0$. If $\lambda_{1} \geqq \lambda_{2} \geqq$ are the eigenvalues of $W$, then $\operatorname{Tr} W^{2}=\sum \lambda_{i}^{2}$ and $\operatorname{Tr} W=\sum \lambda_{i}$. But $(d-1) \sum_{2}^{d} \lambda_{i}^{2} \geqq\left(\sum_{2}^{d} \lambda_{i}\right)^{2}=\lambda_{1}^{2}$, and hence $(d-1) \operatorname{Tr} W^{2} \geqq d \lambda_{1}^{2}$. In addition to the condition $\operatorname{div} v=0, \mathscr{H}$ is supposed to be restricted to the space of divergenceless functions. This restriction might improve (1) but, as in [1], it will not be used here.

The domain $\Omega \in \mathbb{R}^{d}$ is assumed to be an open set of finite volume $|\Omega|$; boundedness is not required. Condition (4) insures that the quadratic form on $H_{0}^{1}(\Omega)$, defined by

$$
Q(\phi, \phi)=v \int|\nabla \phi|^{2}-\int w \phi^{2},
$$

is bounded below and thus defines $H$ as a self-adjoint operator. (Integrals, here and henceforth, are over $\Omega$.) For our purposes, self-adjointness is not important; the only important consideration is the $\max -\min$ principle which can be used as a definition of the $e_{i}$ :

$$
E_{n}=\inf _{\phi} \sum_{i=1}^{n} Q\left(\phi_{i}, \phi_{i}\right)
$$

where $\phi=\left\{\phi_{1}, \ldots, \phi_{n}\right\}$ is any $L^{2}$ orthonormal set in $H_{0}^{1}(\Omega)$. It is, in fact, the right side of (6) that enters in the derivation of the bound (1). 
The goal is to find upper bounds on the following two quantities

$$
\begin{gathered}
E(\gamma) \equiv \sum_{e_{i} \leqq 0}\left|e_{i}\right|^{\gamma}, \quad \gamma \geqq 0, \\
E_{n} \text { itself for fixed } n .
\end{gathered}
$$

It is a consequence of (1) that for $\gamma \geqq 1$

$$
\sum_{\mu_{i} \geqq 0} \mu_{i}^{\gamma} \leqq d \sum_{\left\langle e_{i}\right\rangle \leqq 0}\left|\left\langle e_{i}\right\rangle\right|^{\gamma} \leqq d\langle E(\gamma)\rangle .
$$

This is Karamata's theorem which, more generally, states that when $f: \mathbb{R} \rightarrow \mathbb{R}$ is convex and non-decreasing then (1) implies that

$$
\sum_{i=1}^{n d} f\left(\mu_{i}\right) \leqq d \sum_{i=1}^{n} f\left(-\left\langle e_{i}\right\rangle\right) \leqq d\left\langle\sum_{i=1}^{n} f\left(-e_{i}\right)\right\rangle
$$

for all $n$. If, in addition, $f(t)=0$ for $t \leqq 0$, then

$$
\sum_{\mu_{i} \geqq 0} f\left(\mu_{i}\right) \leqq d \sum_{\left\langle e_{i}\right\rangle \leqq 0} f\left(-\left\langle e_{i}\right\rangle\right) \leqq d\left\langle\sum_{e_{i} \leqq 0} f\left(-e_{i}\right)\right\rangle .
$$

[Actually, Karamata's inequality gives the left-hand inequalities in (9)-(11). The right-hand inequalities come from Jensen's inequality $\langle f(a)\rangle \geqq f(\langle a\rangle)$.]

It is (9) that gives information about the magnitude of the $\mu_{i}$. The bound used in [1] [except for the factor $(d-1) / d$ in (3)] was

$$
E(\gamma) \leqq L_{\gamma, d} v^{-d / 2} \int w(x)^{\gamma+d / 2} d x
$$

The present knowledge about (12) is the following:

(1) $L_{\gamma, d}<\infty$ for $\gamma>\frac{1}{2}(d=1), \gamma>0(d=2), \gamma \geqq 0(d \geqq 3)$. No such bound exists for $\gamma<\frac{1}{2}(d=1)$ or $\gamma=0(d=2)$. The case $\gamma=\frac{1}{2}, d=1$ does not seem to have been settled. (The claim in [2] that $L_{1 / 2,1}<\infty$ is not justified.) Bounds on $L_{1,3}$ were first given by Lieb and Thirring in [3] and on $L_{\gamma, d}$ for $\gamma>0(d=2,3)$ and $\gamma>\frac{1}{2}(d=1)$ in [2]. Bounds on $L_{0, d}, d \geqq 3$, were first given by Cwikel [4], Lieb [5, 6], and Rosenbljum [7]. The best upper bound for $L_{0,3}$ is in [6], namely $0.0780=4 \pi^{-2} 3^{-3 / 2} \leqq L_{0,3}$ $\leqq 0.1156$. The lower bound is from [2, Eq. (4.24)]. Recently, by a simpler method, $\mathrm{Li}$ and Yau [8] derived upper bounds for $L_{0, d}, d \geqq 3$ which they claimed was better than that in [6]; unfortunately a numerical error was made in [8] and their bound for $L_{0,3}$ is three times larger than that in [6].

(2) The sharp constant $L_{\gamma, d}$ in (12) cannot depend on $\Omega$, i.e. $L_{\gamma, d}(\Omega)=L_{\gamma, d}\left(\mathbb{R}^{d}\right)$. To see this, assume that $0 \in \Omega$ and, given $w$ on $\mathbb{R}^{d}$, consider $w_{c}(x)=c^{2} w(c x)$ on $\Omega$. Then let $c \rightarrow \infty$. This situation is in contrast with the $|\Omega|$ dependent bound for $E_{n}$ to be derived later.

(3) There is a natural "guess" for $L_{\gamma, d}$ given by the semiclassical formula

$$
E(\gamma) \approx(2 \pi)^{-d} \iint d p d x\left|v p^{2}-w(x)\right|_{-}^{\gamma}=L_{\gamma, d}^{c} v^{-d / 2} \int w(x)^{\gamma+d / 2} d x
$$

with $|a|_{-}=\max (0,-a)$. An easy integration gives

$$
L_{\gamma, d}^{c}=2^{-d} \pi^{-d / 2} \Gamma(\gamma+1) / \Gamma(\gamma+1+d / 2) .
$$

(4) It is a fact [2] that

$$
L_{\gamma, d} \geqq L_{\gamma, d}^{c} .
$$


In $[2,3]$ it was conjectured that $L_{1, d}=L_{1, d}^{c}$ for $d \geqq 3$. It is known [2] that for each $d \leqq 7$ there is a $\gamma_{d}>0$ such that $L_{\gamma, d}>L_{\gamma, d}^{c}$ when $\gamma<\gamma_{d}$. When $d=1$ or $2, \gamma_{d}>1$. It is also known [9] that $L_{\gamma, 1}=L_{\gamma, 1}^{c}$ for $\gamma \geqq 3 / 2$. In fact [9] the ratio $R_{d}(\gamma)=L_{\gamma, d} / L_{\gamma, d}^{c}$ is monotone non-increasing in $\gamma$; thus if $R_{d}\left(\gamma_{0}\right)=1$ for some $\gamma_{0}$, then $R_{d}(\gamma)=1$ for all $\gamma \geqq \gamma_{0}$. Glaser et al. [10] have shown that $L_{0, d}>L_{0, d}^{c}$ for $d \geqq 7$. They also evaluate $L_{0,4}$ exactly (it is a Sobolev constant) provided $w$ is restricted to be spherically symmetric. For related results see [11].

(5) Inequality (12) for $\gamma=1$ is equivalent [2] to

$$
\sum_{i=1}^{n} \int\left|\nabla \phi_{i}(x)\right|^{2} d x \geqq K_{d} \int \varrho_{\phi}(x)^{1+2 / d} d x,
$$

where the $\left\{\phi_{i}\right\}$ is any $L^{2}$ orthonormal set in $H^{1}\left(\mathbb{R}^{d}\right)\left[\right.$ or $\left.H_{0}^{1}(\Omega)\right]$ and

$$
\varrho_{\phi}(x)=\sum_{i=1}^{n}\left|\phi_{i}(x)\right|^{2} .
$$

The sharp constants in (12) and (16) are related by

$$
L_{1, d}=\left[d / 2 K_{d}\right]^{d / 2}(1+d / 2)^{-1-d / 2} .
$$

[Note: If $n$ is specified then the sharp constant in (16) may depend on $n$, i.e. $K_{d}(n)$. $K_{d}$, the sharp constant in (16), (18) is defined to be $\sup _{n} K_{d}(n)$.] Corresponding to $L_{1, d}^{c}$ in (14) there is a classical value $K_{d}^{c}$ given by (18):

$$
K_{d}^{c}=4 \pi d \Gamma(1+d / 2)^{2 / d} /(2+d) \text {. }
$$

By (15), $K_{d} \leqq K_{d}^{c}$.

An inequality related to (16), and which will be used later in the event that $K_{d}<K_{d}^{c}$, was proved by Li and Yau [8]. It depends on the volume $|\Omega|$ and it is the relation obtained by setting $K_{d}=K_{d}^{c}$ and $\varrho(x)=n /|\Omega|$ in (16). For any orthonormal set in $H_{0}^{1}(\Omega)$

$$
\sum_{i=1}^{n} \int\left|\nabla \phi_{i}(x)\right|^{2} d x>K_{d}^{c} n^{1+2 / d}|\Omega|^{-2 / d} .
$$

(The strict inequality in (20) is, in fact, implied by the proof in [8].)

Before turning to our estimate for $E_{n}$ let us make a few additional remarks about (12).

( $\alpha$ ) Combining (3), (9), (12) we see that the right side of (12) is suitable for passing to the "infinite volume" limit, i.e. in some vague sense it is proportional to the volume. The upper bound we shall obtain later for the quantity introduced in [1],

$$
N(w)=\text { smallest } n \text { such that } E_{n}>0,
$$

will also have this extensivity property. By $(1), d N(w)$ is related to number of nonnegative characteristic exponents and an upper bound on $N(w)$ will yield a bound on the number of non-negative characteristic exponents [see (43)].

$(\beta)$ The bound on $N(w)$ in [1] relied on the fact that $L_{0, d}<\infty$ (which is true if and only if $d \geqq 3$ ) and on the conjecture that $L_{1, d}<L_{0, d}^{c}$. While $L_{0, d}^{c} / L_{1, d}^{c}=1$ $+d / 2>1$, the best bound published so far [6] for $L_{1,3}$ is

$$
L_{1,3} \leqq(6.844) L_{1,3}^{c}=0.04624,
$$

and this exceeds $L_{0,3}^{c}=0.01689$. However, the bound can be improved slightly to 0.04030 [see (51) below]. 
$(\gamma)$ Inequality (12) can be used to derive a lower bound for each $e_{n}$. If $e_{n}(V)$ is the $n^{\text {th }}$ eigenvalue for the potential $V$ in place of $-w$ in (2) then, for any number $e$, it is clear that $e_{n}(-w) \geqq e_{n}\left(-(w+e)_{+}\right)+e$. Take $\gamma=0$ in (12) and set $e=e_{n}$. Then the number of non-positive eigenvalues for $V=-\left(w+e_{n}\right)_{+}$is at least $n$, and (12) yields

$$
n \leqq L_{0, d} v^{-d / 2} \int\left(w(x)+e_{n}\right)_{+}^{d / 2} d x
$$

The integral on the right side of (23) is finite if $e_{n}<0$ or if $|\Omega|$ is finite. It is also monotone in $e_{n}$ and thus (23) yields a lower bound for $e_{n}$.

Now we turn to our main goal which is an upper bound for $E_{n}$. Let $\phi_{1}, \ldots, \phi_{n}$ be the eigenfunctions corresponding to $e_{1} \leqq e_{2} \leqq \ldots \leqq e_{n}$. By virtue of (6) and a limiting argument, any approximating orthonormal set such that $\sum_{n}^{n} Q\left(\phi_{i}, \phi_{i}\right)$ $\leqq E_{n}+\varepsilon$ will suffice. $]$ Let $\varrho_{\phi}(x)=\sum_{1}^{n}\left|\phi_{i}(x)\right|^{2}$.

By (6), (16) and with $p=1+2 / d$,

with

$$
E_{n} \geqq F\left(\varrho_{\phi}\right),
$$

$$
F(\varrho) \equiv v K_{d}\|\varrho\|_{p}^{p}-\int w \varrho
$$

which in turn is greater or equal to

Thus,

$$
G(\varrho) \equiv v K_{d}\|\varrho\|_{p}^{p}-\|w\|_{p^{\prime}}\|\varrho\|_{p} .
$$

$$
\begin{aligned}
E_{n} & \geqq \inf \left\{F(\varrho) \mid \int \varrho=n, \varrho(x) \geqq 0\right\} \\
& \geqq \inf \left\{G(\varrho) \mid \int \varrho=n, \varrho(x) \geqq 0\right\} .
\end{aligned}
$$

However, $\|\varrho\|_{p}|\Omega|^{1 / p^{\prime}} \geqq \int \varrho$, and therefore if we define the function $J$, (for $X>0$ ), and $\tilde{E}_{n}$ by

$$
\begin{gathered}
J(X)=v K_{d} X^{p}-\|w\|_{p^{\prime}} X, \\
\tilde{E}_{n}=\inf \left\{\left.J(X)|X \geqq n| \Omega\right|^{-1 / p^{\prime}}\right\},
\end{gathered}
$$

we have that

$$
E_{n}>\tilde{E}_{n}
$$

The strict inequality in (31) is justified by the fact that $\varrho_{\phi}$ cannot satisfy the Hölder inequality after $(28)$, i.e. $\varrho_{\phi}$ cannot be constant in $\Omega$.

[It is left as an exercise, using the fact that $\|\varrho\|_{p} /\|\varrho\|_{1}$ can be made arbitrarily large, that $\tilde{E}_{n}$ is indeed the infimum in (27).]

The minimum in (30) can be computed to be

$$
\begin{aligned}
E_{n}>\tilde{E}_{n} & =J\left(n|\Omega|^{-1 / p^{\prime}}\right), & & n \geqq|\Omega|^{1 / p^{\prime}} X_{0}, \\
& =J\left(X_{0}\right), & & n \leqq|\Omega|^{1 / p^{\prime}} X_{0}
\end{aligned}
$$

where $J^{\prime}\left(X_{0}\right)=0$, namely

$$
p v K_{d} X_{0}^{p-1}=\|w\|_{p^{\prime}}
$$


In particular, if $n|\Omega|^{-1 / p^{\prime}}$ is greater than or equal to the value $X_{1}>0$ such that $J\left(X_{1}\right)=0$, then $E_{n}>0$. Therefore, $N(w)$ defined by (21) satisfies

$$
\begin{aligned}
N(w) & \leqq \mathscr{S}|\Omega|^{1 / p^{\prime}}\left\{\|w\|_{p^{\prime}} / v K_{d}\right\}^{1 /(p-1)} \\
& =\mathscr{S}|\Omega|\left\{\int d x w(x)^{1+d / 2} /|\Omega|\right\}^{d /(2+d)}\left(v K_{d}\right)^{-d / 2} .
\end{aligned}
$$

The symbol $\mathscr{S}$ denotes "the smallest integer $\geqq$."

If $K_{d}<K_{d}^{c}$, the constant in (34), namely $K_{d}^{-d / 2}$, can be improved as follows. Let $0<\delta<1$ and split the kinetic energy term in two parts using (16) and (20). Thus,

$$
E_{n} \geqq F_{\delta}\left(\varrho_{\phi}\right),
$$

with

$$
F_{\delta}(\varrho)=(1-\delta) v K_{d}^{c} n^{p}|\Omega|^{1-p}+\delta v K_{d}\|\varrho\|_{p}^{p}-\int w \varrho .
$$

As before,

$$
E_{n}>\tilde{E}_{n}(\delta)=(1-\delta) v K_{d}^{c} n^{p}|\Omega|^{1-p}+\inf \left\{\delta v K_{d} X^{p}-\left.\|w\|_{p^{\prime}} X|X \geqq n| \Omega\right|^{-1 / p^{\prime}}\right\} .
$$

Previously, in (32), we discussed the inf in (37). Thus $\tilde{E}_{n}(\delta) \geqq 0$ if $n$ satisfies the following two conditions :

$$
\begin{gathered}
n \geqq|\Omega|^{1 / p^{\prime}} X_{0} \delta^{-1 /(p-1)}, \quad[\operatorname{see}(32),(33)], \\
n^{p-1} v|\Omega|^{1-p}\left\{(1-\delta) K_{d}^{c}+\delta K_{d}\right\} \geqq\|w\|_{p^{\prime}}|\Omega|^{-1 / p^{\prime}} .
\end{gathered}
$$

Condition (39) implies that $\tilde{E}_{n}(\delta) \geqq 0$, provided (38) is satisfied. Choose $\delta$ so that (38) and (39) are the same, namely

$$
\delta=K_{d}^{c}\left[2 K_{d} / d+K_{d}^{c}\right]^{-1} .
$$

Inserting this in (37), we have as before

$$
\begin{gathered}
N(w) \leqq \mathscr{S} A_{d}|\Omega| v^{-d / 2}\left\{\int d x w(x)^{1+d / 2} /|\Omega|\right\}^{d /(d+2)}, \\
\left(A_{d}\right)^{2 / d}=\left[2 K_{d}+d K_{d}^{c}\right]\left[(d+2) K_{d} K_{d}^{c}\right]^{-1} .
\end{gathered}
$$

The inequality (40), (41) is our main result.

We now wish to relate (40), (41) to the turbulence problem, i.e. we want to find an upper bound to

$$
\tilde{N}(\mu) \equiv \text { smallest } n \text { such that } \sum_{i=1}^{n} \mu_{i}<0 \text {. }
$$

By (1),

$$
\begin{aligned}
\tilde{N}(\mu) & \leqq d\left\{\text { smallest integer such that }\left\langle E_{n}\right\rangle>0\right\} \\
& \leqq d\left\{\text { smallest integer such that }\left\langle\hat{E}_{n}\right\rangle \geqq 0\right\},
\end{aligned}
$$

where, for each $w$,

$$
\hat{E}_{n} \equiv \sup \left\{\tilde{E}_{n}(\delta) \mid 0 \leqq \delta \leqq 1\right\}
$$

For each fixed $n$ and $\delta, \tilde{E}_{n}(\delta)$, and $\tilde{E}_{n}$ are functions of $t \equiv\|w\|_{p^{\prime}}^{p^{\prime}}$. Denote them by $\tilde{E}_{n}(\delta, t)$ and $\tilde{E}_{n}(t)$. Direct calculation using $(32)$, shows that $\tilde{E}_{n}(t)$ is a convex function of $t\left(\right.$ not $\left.t^{1 / p^{\prime}}=\|w\|_{p^{\prime}}\right)$. Since $\tilde{E}_{n}(\delta, t)$ differs from $\tilde{E}_{n}(t)$ in a trivial way, $\tilde{E}_{n}(\delta, t)$ is also a convex function of $t$. Since $\hat{E}_{n}(t)$ is the supremum of convex functions, is too is convex in $t$. By Jensen's inequality $\left\langle\hat{E}_{n}\right\rangle \geqq \hat{E}_{n}(\langle t\rangle)$. 
Thus, by expressing the right side of (40) in terms of $\|w\|_{p^{\prime}}^{p^{\prime}}$ and then averaging with respect to $\varrho(d v)$ we obtain the bound sought in [1]:

$$
\tilde{N}(\mu) \leqq \mathscr{S} d A_{d}|\Omega| v^{-d / 2}\left\{\iint \varrho(d v) d x w_{v}(x)^{1+d / 2} /|\Omega|\right\}^{d /(d+2)} .
$$

Finally, let us record some available information about the constants in (41). Using (19) we have

$$
\begin{aligned}
& K_{1}^{c}=\pi^{2} / 3=3.290, \\
& K_{2}^{c}=2 \pi=6.283, \\
& K_{3}^{c}=3\left(6 \pi^{2}\right)^{2 / 3} / 5=9.116 .
\end{aligned}
$$

To bound $K_{d}$ a bound on $L_{1, d}$ is needed.

$d=1$ : The bound in [2, Eq. (2.11)] with $m=1, n=1$ is

$$
L_{1,1} \leqq(4 \pi)^{-1 / 2} \Gamma(5 / 2)^{-1} \Gamma(1 / 2)^{2}(1 / 2)^{-1}=4 / 3 .
$$

$d=2,3$ : In this case we use the formula [6]

$$
\sum_{e_{i} \leqq 0}\left|e_{i}\right|^{\gamma}=\gamma \int_{-\infty}^{0}|e|^{\gamma-1} N_{e} d e,
$$

where $N_{e}$ is the number of eigenvalues of $H \leqq e$. In [6] it is shown (with $v=1$ ) that

$$
N_{e} \leqq(4 \pi)^{-d / 2} \int d x \int_{0}^{\infty} d t t^{-1-d / 2} e^{e t} f(t w(x))
$$

with $f(t)=\max \{0, b(t-a)\}$ and

$$
1 / b=\int_{a}^{\infty}(1-a / y) e^{-y} d y .
$$

Inserting (47) in (46), then doing the $e$ integration, then the $t$ integration [after a change of variable to $t w(x)]$ and finally the $x$ integration, one finds

$$
L_{\gamma, d} \leqq b(4 \pi)^{-d / 2} \Gamma(\gamma+1)(d / 2-1+\gamma)^{-1}(d / 2+\gamma)^{-1} a^{1-d / 2-\gamma} .
$$

The optimum constant $a$ satisfies

$$
a e^{a} \int_{1}^{\infty} e^{-a y} d y / y=(d / 2+\gamma-1) /(d / 2+\gamma)
$$

When $\gamma=1$ we take $a=0.61, b=3.6807$ for $d=2$ and $a=1.02, b=6.9358$ for $d=3$. Inserting this in (49) yields

$$
\begin{aligned}
& L_{1,2} \leqq 0.24008, \\
& L_{1,3} \leqq 0.040304 .
\end{aligned}
$$

Using (18)

$$
\begin{aligned}
& K_{1} \geqq 1 / 12=0.08333, \\
& K_{2} \geqq 1.0413, \\
& K_{3} \geqq 2.7709,
\end{aligned}
$$


which, by (41), leads to

$$
\begin{aligned}
& A_{1}=2.050, \\
& A_{2}=0.5597, \\
& A_{3}=0.1329 .
\end{aligned}
$$

This value for $A_{3}$ can be compared with the value in [1, Footnote 7], which is obtained under the conjectured assumptions $L_{0,3}=0.0780$ and $L_{1,3}=L_{1,3}^{c}$, namely

$$
L_{0,3}\left[1-\left(L_{1,3}^{c} / L_{0,3}^{c}\right)^{2 / 5}\right]^{-3 / 2}=0.459 .
$$

If $K_{3}=K_{3}^{c}$, which is conjectured to be true, (41) yields

$$
A_{3}=\left(K_{3}^{c}\right)^{-3 / 2}=0.03633 \text {. }
$$

In addition to the improvement in (53) over (54), we also note the additional factor $(d-1) / d$ in $(3)$ which yields a factor $[(d-1) / d]^{d / 4}$ when the right sides of $(40)$, (43) are expressed in terms of $\varepsilon(x)$. This factor is 0.7378 for $d=3$ and 0.7071 for $d=2$.

Acknowledgement. I should like to thank David Ruelle for stimulating this work and for several helpful conversations.

\section{References}

1. Ruelle, D.: Large volume limit of the distribution of characteristic exponents in turbulence. Commun. Math. Phys. 87, 287-302 (1982)

2. Lieb, E., Thirring, W.: Inequalities for the moments of the eigenvalues of the Schrödinger Hamiltonian and their relation to Sobolev inequalities. In: Studies in mathematical physics: essays in honor of Valentine Bargmann, Lieb, E., Simon, B., Wightman, A. (eds.), pp. 269-303. Princeton, $\mathrm{NJ}$ : Princeton University Press 1976

3. Lieb, E., Thirring, W.: Bound for the kinetic energy of fermions which proves the stability of matter. Phys. Rev. Lett. 35, 687-689 (1975); 35, 1116 (1975) (Erratum)

4. Cwikel, M.: Weak type estimates for singular values and the number of bound states of Schrödinger operators. Ann. Math. 106, 93-100 (1977)

5. Lieb, E.: Bounds on the eigenvalues of the Laplace and Schrödinger operators. Bull. Am. Math. Soc. 82, 751-753 (1976); the details appear in [6]

6. Lieb, E.: The number of bound states of one-body Schrödinger operators and the Weyl problem. Proc. Am. Math. Soc. Symp. in Pure Math., Osserman, R., Weinstein, A. (eds.), Vol. 36, pp. 241-252 (1980). Much of this material is reviewed in Simon, B.: Functional integration and quantum physics, pp. 88-100. New York: Academic Press 1979

7. Rosenbljum, G.: Distribution of the discrete spectrum of singular differential operators. Dokl. Akad. Nauk SSSR 202, 1012-1015 (1972) (MR 45 No. 4216). The details are given in: Distribution of the discrete spectrum of singular differential operators. Izv. Vyss. Ucebn. Zaved. Matem. 164, 75-86 (1976) [English transl. Sov. Math. (Iz. VUZ) 20, 63-71 (1976)]

8. Li, P., Yau, S.-T.: On the Schrödinger equation and the eigenvalue problem. Commun. Math. Phys. 88, 309-318 (1983)

9. Aizenman, M., Lieb, E.: On semiclassical bounds for eigenvalues of Schrödinger operators. Phys. Lett. 66 A, 427-429 (1978)

10. Glaser, V., Grosse, H., Martin, A.: Bounds on the number of eigenvalues of the Schrödinger operator. Commun. Math. Phys. 59, 197-212 (1978)

11. Grosse, H.: Quasiclassical estimates on moments of the energy levels. Acta Phys. Austr. 52, 89-105 (1980)

Communicated by A. Jaffe

Received October 21, 1983 